\title{
Health Assessment of Large Two Dimensional Structures Using Limited Information: Recent Advances
}

\author{
Ajoy Kumar Das, ${ }^{1}$ Achintya Haldar, ${ }^{1}$ and Subrata Chakraborty ${ }^{2}$ \\ ${ }^{1}$ Department of Civil Engineering and Engineering Mechanics, University of Arizona, P.O. Box 210072, Tucson, AZ 85721, USA \\ ${ }^{2}$ Department of Civil Engineering, Bengal Engineering and Science University, Howrah, 711103 WB, India
}

Correspondence should be addressed to Achintya Haldar, haldar@u.arizona.edu

Received 2 March 2011; Revised 24 May 2011; Accepted 8 June 2011

Academic Editor: Alessandro Marzani

Copyright (C) 2012 Ajoy Kumar Das et al. This is an open access article distributed under the Creative Commons Attribution License, which permits unrestricted use, distribution, and reproduction in any medium, provided the original work is properly cited.

Some recent advances of a recently developed structural health assessment procedure proposed by the research team at the University of Arizona, commonly known as generalized iterative least-squares extended Kalman filter with unknown input (GILSEKF-UI) are presented. The procedure is a finite elements-based time-domain system-identification technique. It can assess structural health at the element level using only limited number of noise-contaminated responses. With the help of examples, it is demonstrated that the structure can be excited by multiple loadings simultaneously. The method can identify defects in various stages of degradation in single or multiple members and also relatively less severe defect. The defective element(s) need not be in the substructure, but the defect detection capability increases if the defect spot is close to the substructure. Two alternatives are suggested to locate defect spot more accurately within a defective element. The paper advances several areas of GILS-EKF-UI to assess health of large structural systems.

\section{Introduction}

Fully functional infrastructure is essential for the economic well-being of a city, a state, a country, or a region. As expected, investment in infrastructure has been growing enormously over the years. Globally, an estimate of $2 \%$ of gross domestic product (GDP), about US $\$ 960$ billion, is spent annually on infrastructure investment and maintenance [1]. Global infrastructure expenditure is expected to reach $\$ 35$ trillion over the next 20 years [2]. Based on the Report Card of 2005 for Americas Infrastructure [3], it was estimated that over $\$ 1.6$ trillion over a five-year period was necessary to bring the nation's infrastructure to a good condition. Most civil infrastructure systems are designed for finite design life, since they are exposed to elements and deteriorate with time. Ideally, they should be replaced soon after the expiration of their design life. Unfortunately, most of the countries do not have enough financial resources to replace them. Even in cases where resources are available, it may not be possible to replace them all at the same time; a priority list of their replacement needs to be developed.
However, if they are maintained properly, their performance and operating life can be extended significantly, and this approach is expected to be much better alternative than replacement. Thus, the major challenge to the profession at present is how to assess their current health for making appropriate maintenance decision. This is one of the major forces behind the growth of a multidisciplinary research area, commonly referred to as the structural health assessment (SHA) and maintenance.

Issues related to SHA are very common in standards and design guidelines. However, since inspection costs money and some inconvenience to the users, the major thrust of SHA has been limited to visual inspections at a regular interval (every two years for most bridges) or just after major natural or man-made destructive events. It has been established that visual inspection is inadequate to assess overall structural integrity; its effectiveness depends on the qualification and experience of inspectors, locations and types of defects, and accessibility of the defect spots. Inspection of a bridge over a river or a multistory building during its normal operating hours could be very challenging 
and expensive. For an example, numerous welds fractured in moment-resisting steel frames during the Northridge earthquake of 1994. Similar welds also fractured during the Loma Prieta earthquake of 1989 but remained undetected during this period of about five years. It has also been concluded by the profession that nondestructive inspection (NDI) procedures produce better results when the health is assessed based on current structural behavior rather than other analytical evaluation techniques or visual inspection procedures. This led to the development of several NDI methods using measured response information. They can be broadly classified into two groups: nonmodel and modelbased $[4,5]$. Nonmodel-based techniques rely on the processing of measured quantities or responses without taking into account the mathematical description of the monitored structure. On the other hand, model-based techniques attempt to track changes in the parameters of mathematical model being used to represent the structure. Nonmodel-based methods include visual inspections, listening to audible variations of responses due to tapping of structural surface, imaging structures using X-ray, gamma ray, measuring state of stress using guided wave or eddy current to locate cracks, and so forth. Due to the rapid advancement in data-processing technologies, the changes in structural behavior can also be established by measuring traveling time of signal through structural components, identifying presence of spikes or impulses in time-frequency representation of a signal using wavelet transform (WT) or Hilbert-Huang transform (HHT).

Methods using change in modal properties (natural frequencies, mode shape, and damping ratio) can also be classified as nonmodel-based [5]. Modal properties are global properties; they may identify defects in the overall sense but not at the local element level. Alternatively, changes in the mode shape curvature and modal strain energy can be used to detect damage. Model updating methods, which map the modal properties of an analytical model to the modal properties of the measured model for structural damage detection, are also used. To relax the mapping of analytical frequency and mode shape values of every mode to those of the synthesized model and to provide more information within a desired frequency range, frequency response function are employed as updating variables.

Model-based methods were developed to overcome the deficiencies of nonmodel-based methods. The aim of a model-based SI technique is to predict the parameters of the assumed mathematical model of a physical system; in other words, the system is considered to behave in predetermined manner represented in algorithmic form using the governing differential equations, finite element (FE) discretization, and so on. Because of its simplicity, the model-based methods were developed initially using measured response information caused by the static application of the loadings. There are several advantages to this approach including that the amount of data needed to be stored is relatively small and simple and no assumption on the mass or damping characteristics is required. Thus, less errors and uncertainties are introduced into the model. However, there are several disadvantages including that the number of measurement points should be larger than the number of unknown parameters to assure a proper solution. Civil engineering structures are generally large and complex with extremely high overall stiffness. It may require extremely large static load to obtain measurable deflections. Fixed reference locations are required to measure deflections which might be impractical to implement for bridges, offshore platforms, and so forth. Also, static response-based methods are sensitive to measurement errors [5].

Methods based on measured dynamic responses have many advantages including that it is possible to excite the structures by dynamic loadings of small amplitude relative to static loadings. In some cases, ambient responses cased by natural sources such as wind, earthquake, moving vehicle, and so forth, can also be used. If acceleration responses are measured, they eliminate the need for fixed physical reference required for measurement of deflections. They perform well even in the presence of higher level of measurement errors.

For the SHA of civil infrastructure systems, the most efficient use of the available limited resources will be to detect defects and their severity at the local element level (in a beam or column or within a segment of a defective beam or column). This will necessitate the FE representation of the system. After an exhaustive literature review, the research team at the University of Arizona concluded that if a dynamic system is represented by FEs and responses are available in time domain, by tracking the changes in the stiffness parameter of the elements, the location and severity of defects can be established. This will necessitate the use of a system identification (SI)-based approach. A basic SI-based approach has three essential components: (a) the excitation force(s), (b) the system to be identified, generally represented by some equations in algorithmic form such as by FEs, and (c) the output response information measured by sensors. Using the excitation and response information, the third component, the system can be identified.

Outside the controlled laboratory environment, measuring input excitation force(s) can be very expensive and problematic during health assessment of a real existing structure. In the context of an SI-based approach, it will be desirable if a system can be identified using only measured response information, and completely ignoring the excitation information. This task is expected to be challenging, since two of the three basic components of SI process will be unknown. Following this thought process, the research team sequentially proposed several methods including iterative least-squares with unknown input (ILS-UI) [6], modified iterative least-squares with unknown input (MILS-UI) [7], and generalized iterative least-squares with unknown input (GILS-UI) [8]. The major weakness of these procedures is that they require dynamic response information at all dynamic degrees of freedom (DDOFs). Measuring dynamic response information at all DDOFs of large complicated civil infrastructure may not be practicable and is expected to be economically prohibitive. Furthermore, measured response information is expected to contain noise even when they are recorded by smart sensors. As will be discussed later, the team then proposed an extended Kalman filter (EKF)-based SI approach addressing all major related issues. 
The basic concept is very novel and is being developed in stages to increase its implementation potential and to address suggestions made by other researchers closely following the developments. Some of the recent developments are presented in this paper.

For the sake of completeness of this discussion, available SHA procedures are classified into four levels as discussed in [9]. They are: Level 1-determination if damage is present in a structure, Level 2-determination of geometric location of the damage, Level 3-assessment of severity of the damage, and Level 4-prediction of remaining life of the structure. The method discussed in this paper will address Levels 1, 2, and 3.

It is not possible to refer to all major works in the related areas. Reviews of the current state-of-the-art of vibration-based SI procedures can be found in $[5,10-21]$. Some interesting philosophies regarding structural health monitoring (SHM) and possible new directions are suggested in [22-24]. Worden et al. [25] discussed certain fundamental axioms of SHM. Friswell [26] presented an overview of inverse problem for structural damage identification based on vibration responses. The data processing necessary for intelligent damage identification can be considered either as damage identification in an inverse problem or damage identification in pattern recognition problem [22].

\section{Applications of KF and EKF for Structural Health Assessment (SHA): A Brief Review}

As discussed earlier, the essential building block of the method presented here is a Kalman filter (KF)-based SI procedure. The reasons for its use need further elaboration since a typical civil engineer may not be familiar with them. The basic problem is that deterministic mathematical models and control theories do not appropriately represent the behavior of a physical system, and thus the SI-based method may not be appropriate. Maybeck [27] correctly pointed out three basic reasons behind this: (i) no mathematical model to represent a system is perfect, (ii) dynamic systems are not only driven by control inputs, but there are always disturbances that cannot be controlled and modeled deterministically, and (iii) the responses observed by sensors do not exhibit the actual perfect system responses since sensors always introduce their own system dynamics and distortion into the measured data. Thus, the procedures must account for different sources of uncertainty, and the system parameters need to be evaluated in an optimal sense using proper data processing algorithm. KF-based algorithms are ideal and widely used for nondeterministic analysis in science and engineering.

Kalman filter $[28,29]$ is a set of mathematical equations that provides efficient computational means in a recursive manner to estimate the state of a process in a way that minimizes the mean of squared error and calculates the best estimate of states from the noisy sensor responses [30-33]. It is a time-domain filter and is very powerful in several aspects. It supports estimations of past, present, and even future states, and it can do so even when the precise nature of the modeled system is unknown. It incorporates the (i) knowledge of the system, (ii) statistical description of the system noises, measurement errors, and uncertainty in the dynamic models, and (iii) any available information on the initial conditions of the variables of interest [27].

In a mathematical sense, KF addresses the general problem of trying to estimate the state of a discrete-time controlled process that is governed by a linear stochastic differential equation. In actual applications, the process to be estimated and/or the measurement relationship to the process may not be linear. For example, responses observed for SHA of civil infrastructure are, in general, not linear. The presence of defects can also make the responses nonlinear. The EKF $[34,35]$ concept was introduced to address mildly nonlinear behavior. It is an extension of KF that linearizes about the current mean and covariance. However, the level of nonlinearity when it will not work is not yet established in the literature and is now under active consideration by the team.

Many SHA procedures available in the literature were developed considering different sources of uncertainties to optimally identify the structural system parameters using basic KF concept [36-38] and EKF concept [11, 12, 36, 3941]. As will be discussed mathematically later, to implement these concepts, time history of responses measured at equal interval for a period of time is used, giving a total of say $m$ data points. The model parameters are estimated and updated at each time instance for a total of $m$ times, known as the local iteration. When the process is repeated for the whole duration of response for $m$ times, it is generally known as the completion of the first global iteration. If the parameters of system do not converge with a predetermined convergence, the global iterations need to be repeated. In order to improve the efficiency considering stability and convergence, Hoshiya and Saito [42] proposed weighted global iteration (WGI) [30] procedure with an objective function in the EKF method. The procedure was verified extensively by identifying structural parameters [42-48].

In many situations, complete structural identification may not be required, particularly, when the region that contains defect is known as a priori. A localized structural identification procedure, often referred to as substructuring approach [46-48], would serve the purpose. The localized substructuring procedure has the advantage that identification of input excitation is not necessary if the node of excitation does not belong to the substructure under consideration. However, multiple substructures will be necessary for complete structural identification.

Recently, certain aspects of EKF procedure have been considered for improvement by several authors. Koh and See [49] proposed an adaptive EKF (AEKF) to estimate both the parameter values and associated uncertainties in identification. The procedure works well both without and with presence of modeling error. They verified the procedure using simulation results. Recently, Yang et al. [50] proposed an adaptive tracking technique based on EKF to identify structural parameters and their variation during damage events. The procedure was verified using simulation [50, 51] and experimental results [52]. Hoshiya and Sutoh [53] 
proposed an FE-based EKF with weighted local iteration (EKF-WLI) and verified for elastic plane strain field problem in geotechnical engineering. Ghosh et al. [54] developed two novel forms of EKF-based parameter identification techniques; these are based on variants of the derivativefree locally transversal linearization (LTL) and multistep transverse linearization (MTrL) procedures. Liu et al. [55] proposed a KF-based algorithm, called MMAE-WGI-FL MOKF, for the SHA of beam using numerical and experimental responses. The procedure uses a multiple model adaptive estimator (MMAE) that consists of bank of EKF designed in the modal domain (MOKF) and incorporated fuzzy logic-based block in EKF to estimate variance of measurement noise. The algorithm uses a weighted global iterative (WGI) scheme.

As mentioned earlier, the research group at University of Arizona developed several SHA procedures (ILS-UI, MILSUI, and GILS-UI) incorporating all the issues discussed earlier. The team also proposed another method and called it as iterative least-squares extended Kalman filter with unknown input (ILS-EKF-UI) [56]. The basic idea of the procedure is to assess structural health considering uncertainties in the mathematical model and FE representation and using limited numbers of noise contaminated responses measured by smart sensors at a small part of the structure. The concept was extensively analytically verified for simple configuration of defect-free structures using numerically simulated responses [56,57], as is commonly practiced. Later they improved the procedure and proposed the generalized iterative least-squares extended Kalman filter with unknown input (GILS-EKF-UI) [58] method. The procedure was extensively verified using numerically simulated response information for defect-free and defective frames [58-60]. It was then verified by conducting extensive experimental investigations in the laboratory. A one-third scale model of a two-dimensional one-bay three-story steel frame was built in the laboratory. The health assessment capability of the procedure was studied by exciting the frame by sinusoidal and impulsive loadings at the superstructure. Using response information only, the health of the frame was successfully assessed for defect-free and various defective configurations [61-64]. The success of the study (numerical as well as experimental) prompted the research team to extend the concept to assess structural health of more complex and large two- and three-dimensional structures in the presence of several types and severity of defects to establish its wider application potential.

The team already investigated the implementation potential of the method by conducting extensive laboratory investigations on a relatively smaller two-dimensional frame. Laboratory and field investigations of larger frames will require additional enormous amount of financial resources not available at this time. Using the previous experience, the extensions of GILS-EKF-UI are presented here using analytically generated response information. In the past, the team presented additional tasks required to assess structural health using measured response information [61-64]. It is to be noted that most of the past works by other researchers have been conducted using only analytical response information.
Most of the SHA procedures based on KF and EKF cannot be used to assess structural health of existing structures. Some of the implementation issues that require further attention are addressed in this paper. (1) The available SIbased SHA procedures using KF and EKF concepts were essentially developed and verified for simplest forms of structures represented by shear buildings, one dimensional beams, two-dimensional trusses, and frames with relatively small numbers of DDOFs. They need to be verified for systems with larger numbers of DDOFs. (2) The issues related to measurement and/or identification of input excitation is not addressed. This adds a major degree of complexity and often overlooked by assuming it is known or can be accurately measured, for example, highly irregular seismic ground motion. (3) The procedures other than the inverse identification techniques, referred to as pattern recognition procedures, try to identify defect by identifying certain pattern or trend by using signal processing techniques; they do not explicitly consider mathematical model of the structural dynamics and cannot quantify severity of defects. (4) In general, the determination of location(s) of defect(s), their severity, and implication of structural integrity are not addressed in most of the available procedures. (5) For large complex structures, the application of the procedure may not be straight forward. Several issues including selection of substructure(s), excitation by multiple forces, types and severity of defects, location of several defects in multiple members, and their location with respect to the substructure(s), identification of defect spot more accurately within a defective member, and so forth need further elaboration. There are several other factors related to the numerical algorithm including weight factor, convergence criteria, location and types of excitation (superstructure or at the base), optimum number of responses required to assess large structural systems, and so forth also need further attention. Some of the extensions are presented in this paper with the help of a relatively larger frame.

\section{The GILS-EKF-UI Method}

Before presenting the GILS-EKF-UI procedure, it may be informative to discuss how it can be implemented for assessing health of real existing structures. Very complicated structural systems will be represented by finite elements. Assuming that the mass is known, the stiffness and damping parameters of all the elements will be identified using only limited number of noise-contaminated acceleration response-time histories measured at some part(s) of the structure using the two-stage GILS-EKF-UI procedure. By tracking changes in the stiffness parameters from the previous, expected, or design values or by comparing with other similar elements, the location(s) and the severity of the defects are established.

To satisfy all the requirements to implement the EKF concept and at the same time to satisfy the dynamic governing equation, the unknown excitation information and the initial state vector must be available. However, they are unavailable at this stage. To address this challenge, 
the research team proposed to combine GILS-UI and EKFWGI approaches in two stages, as discussed next.

Stage 1 (substructure identification). A substructure is a small part of a structure that satisfies all the requirements to implement the GILS-UI procedure. Based on the available measured response information, substructure(s) are selected, as discussed by Katkhuda and Haldar [58]. The size of the substructure should be kept to a minimum for economic reason. Past maintenance history of similar structures, or experience of the inspector can also help in selecting an appropriate substructure. However, the defect predictability improves significantly when the defect is located close to the substructure. This will be discussed later with an example. Multiple substructures may be necessary for large structures since at least one of them will be closer to the location of defect. However, as in many other engineering problems, applying engineering judgment will significantly improve the damage predictability of the procedure. For a relatively large frame structure shown in Figure 1, the substructure used to assess health is shown by the double lines although it may be located at different location of the frame and its configuration could be different. Using the GILS-UI procedure, the stiffness and damping parameters of all the elements in the substructure can be identified. Information on the time history of input excitation(s) that caused the responses will also be generated. The generated information is then used to implement the EKF-WGI method in Stage 2.

Stage 2 (identification of whole structure). The identified stiffness parameters of the substructure can be judiciously used to develop the initial state vector of stiffness parameters for the whole frame, since they are expected to be similar. The excitation information is also known at this stage. Thus, in Stage 2, the health of the whole frame can be assessed using only limited number of noise-contaminated responses using EKF-WGI, resulting in the GILS-EKF-UI procedure.

\section{Mathematical Concept of GILS-EKF-UI}

Mathematical concepts of the two stages are discussed next.

Stage 1 (mathematical concept of GILS-UI method). The governing differential equation of motion using Rayleigh damping for the substructure can be expressed as

$\mathbf{M}_{\text {sub }} \ddot{\mathbf{X}}_{\text {sub }}(t)+\left(\alpha \mathbf{M}_{\text {sub }}+\beta \mathbf{K}_{\text {sub }}\right) \dot{\mathbf{X}}_{\text {sub }}(t)+\mathbf{K}_{\text {sub }} \mathbf{X}_{\text {sub }}(t)=\mathbf{f}_{\text {sub }}(t)$,

where $\mathbf{M}_{\text {sub }}$ is the global mass matrix, $\mathbf{K}_{\text {sub }}$ is the global stiffness matrix, $\ddot{\mathbf{x}}_{\text {sub }}(t), \dot{\mathbf{x}}_{\text {sub }}(t)$, and $\mathbf{x}_{\text {sub }}(t)$ are the vectors containing the acceleration, velocity, and displacement, respectively, at time $t, \mathbf{f}_{\text {sub }}(t)$ is the input excitation vector at time $t$, and $\alpha$ and $\beta$ are the mass and stiffness proportional Rayleigh damping coefficients, respectively. The subscript "sub" is used to denote substructure.
For a two-dimensional frame, the mass matrix $\mathbf{M}_{i}$ and stiffness matrix $\mathbf{K}_{i}$ for $i$ th element in the global coordinate system can be expressed as

$$
\begin{gathered}
\mathbf{M}_{i}=\mathbf{T}_{i}^{T} \overline{\mathbf{M}}_{i} \mathbf{T}_{i}, \\
\mathbf{K}_{i}=\mathbf{T}_{i}^{T} \overline{\mathbf{K}}_{i} \mathbf{T}_{i}=k_{i} \mathbf{S}_{i},
\end{gathered}
$$

where $\mathbf{T}_{i}$ is the $(6 \times 6)$ transformation matrix and written for two-dimensional $i$ th frame element as

$$
\mathbf{T}_{i}=\left[\begin{array}{cccccc}
\cos \theta & \sin \theta & 0 & 0 & 0 & 0 \\
-\sin \theta & \cos \theta & 0 & 0 & 0 & 0 \\
0 & 0 & 1 & 0 & 0 & 0 \\
0 & 0 & 0 & \cos \theta & \sin \theta & 0 \\
0 & 0 & 0 & -\sin \theta & \cos \theta & 0 \\
0 & 0 & 0 & 0 & 0 & 1
\end{array}\right]
$$

$\overline{\mathbf{M}}_{i}$ and $\overline{\mathbf{K}}_{i}$ are mass and stiffness matrix for the ith element of size $(6 \times 6)$ in the local element coordinate system and can be written as

$$
\begin{gathered}
\overline{\mathbf{M}}_{i}=\frac{\bar{m}_{i} L_{i}}{420}\left[\begin{array}{cccccc}
140 & & & & \\
0 & 156 & & & \text { sym. } \\
0 & 22 L_{i} & 4 L_{i}^{2} & & & \\
70 & 0 & 0 & 140 & & \\
0 & 54 & 13 L_{i} & 0 & 156 & \\
0 & -13 L_{i} & -3 L_{i}^{2} & 0 & -22 L_{i} & 4 L_{i}^{2}
\end{array}\right], \\
\overline{\mathbf{K}}_{i}=\frac{E_{i} I_{i}}{L_{i}}\left[\begin{array}{cccccc}
\frac{A_{i}}{I_{i}} & & & & & \\
0 & \frac{12}{L_{i}^{2}} & & & & \\
0 & \frac{6}{L_{i}} & 4 & & & \\
-\frac{A_{i}}{I_{i}} & 0 & 0 & \frac{A_{i}}{I_{i}} & & \\
0 & -\frac{12}{L_{i}^{2}} & -\frac{6}{L_{i}} & 0 & \frac{12}{L_{i}^{2}} & \\
0 & \frac{6}{L_{i}} & 2 & 0 & -\frac{6}{L_{i}} & 4
\end{array}\right]=k_{i} \overline{\mathbf{S}}_{i},
\end{gathered}
$$

where $L_{i}$ is length, $m_{i}$ is mass per unit length, $I_{i}$ is moment of inertia of the cross section, and $E_{i}$ is material elastic modulus for the $i$ th element, $k_{i}$ is the $i$ th element stiffness parameter defined as $E_{i} I_{i} / L_{i}$, and $\overline{\mathbf{S}}_{i}$ is the stiffness coefficients in the square bracket in the local coordinate system. When $\overline{\mathbf{S}}_{i}$ is transformed in the global coordinate system, it becomes a part of $\boldsymbol{S}_{i}$ as in (3). For a substructure containing $N_{\text {sub }}$ number of DDOFs and nesub number of elements, the global 


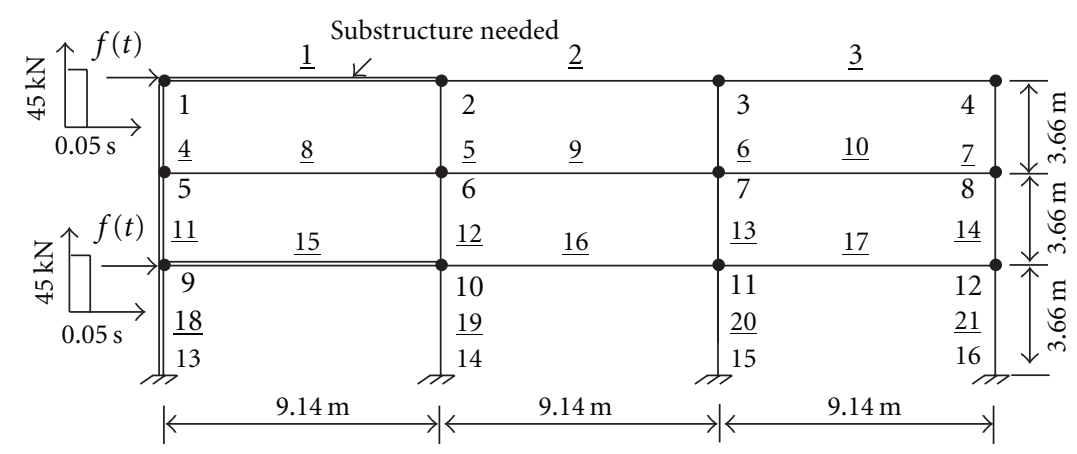

Figure 1: A three-bay three-story frame under impulsive excitation.

mass matrix $\mathbf{M}_{\text {sub }}$ and stiffness matrix $\mathbf{K}_{\text {sub }}$ will have size of $\left(N_{\text {sub }} \times N_{\text {sub }}\right)$, and they can be assembled as

$$
\begin{gathered}
\mathbf{M}_{\text {sub }}=\sum_{i=1}^{n e s u b} \mathbf{M}_{i} \\
\mathbf{K}_{\text {sub }}=\sum_{i=1}^{n e s u b} \mathbf{K}_{i}=\sum_{i=1}^{n e s u b} k_{i} \mathbf{S}_{i}=k_{1} \mathbf{S}_{1}+k_{2} \mathbf{S}_{2}+\cdots+k_{\text {nesub }} \mathbf{S}_{\text {nesub }}
\end{gathered}
$$

As discussed by Katkhuda and Haldar [58], if $N_{d k e y}$ is the number of DDOFs for the key node(s) in the substructure, (1) can be reorganized with $N_{d \text { key }} \times m$ number of equations as

$$
\mathbf{A}_{N d \text { key } \cdot m \times L \text { sub }} \mathbf{P}_{L \text { sub } \times 1}=\mathbf{F}_{N d \text { key } \cdot m \times 1},
$$

where

$$
\mathbf{P}_{L \text { sub } \times 1}=\left[k_{1}, k_{2}, \ldots, k_{\text {nesub }}, \beta k_{1}, \beta k_{2}, \ldots, \beta k_{n e s u b}, \alpha\right]^{T} .
$$

In the overdetermined system of equation represented by (7), A is a rectangular matrix with $N_{d \text { key }} \times m$ rows and $L_{\text {sub }}$ columns $\left(N_{d \text { key }} \times m>L_{\text {sub }}\right)$ and populated with system response vectors of velocity $\dot{\mathbf{x}}_{\text {sub }}$ and displacement $\mathbf{x}_{\text {sub }}$ for all $m$ time points, and $\mathbf{P}$ is a column vector of size $\left(L_{\text {sub }} \times\right.$ 1) containing nesub number of unknown stiffness and two damping parameters $\alpha$ and $\beta$, resulting $L_{\text {sub }}=2 n e s u b+1$, as in (8). F is a column vector of $N_{d k e y} \times m$ rows composed of unknown input excitations and inertia forces for all $m$ time points.

A least-squares-based procedure proposed by Wang and Haldar [6] is used for the solution of unknown system parameters $\mathbf{P}$ using the following expression:

$$
\begin{aligned}
\mathbf{P}_{L \text { sub } \times 1}= & \left(\mathbf{A}_{L \text { sub } \times N d \text { key } \cdot m}^{T} \mathbf{A}_{N d \text { key } \cdot m \times L \text { sub }}\right)^{-1} \mathbf{A}_{L \text { sub } \times N d \text { key } \cdot m}^{T} \\
& \times \mathbf{F}_{N d \text { key } \cdot m \times 1} .
\end{aligned}
$$

The unknown system parameter vector $\mathbf{P}$ can be solved provided the force vector $\mathbf{F}$ and matrix $\mathbf{A}$ are known. However, since the input excitation is not known, vector $\mathbf{P}$ is solved by starting an iteration process by assuming that the unknown input excitation to be zero at all $m$ time points $[8,58]$. This assumption will assure a nonsingular solution of (7), without compromising the convergence or the accuracy of the method. It is observed that the method is not sensitive to this initial assumption, the type and form of excitation [6].

Stage 2 (mathematical concept of EKF-WGI method). For the implementation of EKF procedure, the differential equation in state-space form and the discrete time measurements can be expressed as

$$
\begin{gathered}
\dot{\mathbf{Z}}_{t}=f\left(\mathbf{Z}_{t}, t\right), \\
\mathbf{Y}_{t_{k}}=h\left(\mathbf{Z}_{t_{k}}, t_{k}\right)+\mathbf{V}_{t_{k}},
\end{gathered}
$$

where $\mathbf{Z}_{t}$ is the state vector at time $t, \dot{\mathbf{Z}}_{t}$ is the time derivative of the state vector, $f$ is a nonlinear function of the state, $\mathbf{Y}_{t_{k}}$ is the measurement vector, $h$ is the function that relates the state to the measurement, $\mathbf{V}_{t_{k}}$ is a zero-mean, uncorrelated, white noise process with variance $R_{k}$, and represented by $E\left[V_{t_{k}} V_{t_{j}}^{T}\right]=R_{k} \delta_{k-j}$, where $\delta_{k-j}$ is the Kronecker delta function; that is $\delta_{k-j}=1$ if $k=j$, and $\delta_{k-j}=0$ if $k \neq j$.

For a structure represented by $N$ number of degrees of freedom and ne number of elements, the vectors $\mathbf{Z}_{t}$ and $\dot{\mathbf{Z}}_{t}$, are of size $(2 N+L) \times 1, L$ is the total number of unknown stiffness parameters. They are formed in the following way:

$$
\begin{aligned}
\mathbf{Z}_{t} & =\mathbf{Z}(t)=\left[\begin{array}{c}
\mathbf{Z}_{1}(t) \\
\mathbf{Z}_{2}(t) \\
\mathbf{Z}_{3}(t)
\end{array}\right]=\left[\begin{array}{c}
\mathbf{X}(t) \\
\dot{\mathbf{X}}(t) \\
\tilde{\mathbf{K}}
\end{array}\right], \\
\dot{\mathbf{Z}}_{t} & =\dot{\mathbf{Z}}(t)=\left[\begin{array}{c}
\dot{\mathbf{X}}(t) \\
\ddot{\mathbf{X}}(t) \\
0
\end{array}\right] \\
& =\left[\begin{array}{c}
\dot{\mathbf{X}}(t) \\
-\mathbf{M}^{-1}[\mathbf{K X}(t)+(\alpha \mathbf{M}+\beta \mathbf{K}) \dot{\mathbf{X}}(t)-\mathbf{f}(t)] \\
0
\end{array}\right],
\end{aligned}
$$

where $\tilde{\mathbf{K}}=\left[\begin{array}{lllll}k_{1} & k_{2} & \cdots & k_{n e}\end{array}\right]^{T}$ is column vector of size $(L \times 1)$.

For the identification of the whole structure, acceleration responses will be measured at a fewer $(B)$ number of DDOFs. The accelerations will be integrated twice to obtain 
the velocities and displacements, as described by Vo and Haldar [65]. The vector $\mathbf{Y}_{t_{k}}$ will have size $(2 B \times 1)$ and will contain information on observed displacements and velocities. Information on acceleration will not be necessary for Stage 2.

The filtering process in EKF can be started after initialization of state vector $\mathbf{Z}\left(t_{0} \mid t_{0}\right)$, which can be assumed to be Gaussian random variable with state mean $\widehat{\mathbf{Z}}\left(t_{0} \mid t_{0}\right)$ and error covariance of $\mathbf{P}\left(t_{0} \mid t_{0}\right)$ that is, $\mathbf{Z}\left(t_{0} \mid t_{0}\right) \sim N\left(\hat{\mathbf{Z}}_{t_{0}}, \mathbf{P}_{t_{0}}\right)$.

The initial error covariance matrix $\mathbf{P}\left(t_{0} \mid t_{0}\right)$ contains information on the errors in the observed displacement and velocity responses and in the initial values assigned to the unknown stiffness parameters of the whole structure. It is generally assumed to be diagonal and can be expressed as $[56,58]$

$$
\mathbf{P}\left(t_{0} \mid t_{0}\right)=\left[\begin{array}{cc}
\mathbf{P}_{x}\left(t_{0} \mid t_{0}\right) & 0 \\
0 & \mathbf{P}_{k}\left(t_{0} \mid t_{0}\right)
\end{array}\right],
$$

where $\mathbf{P}_{x}\left(t_{0} \mid t_{0}\right)$ is a $(2 \mathrm{~N} \times 2 \mathrm{~N})$ diagonal matrix contains initial error covariance for observed responses, and $\mathbf{P}_{k}\left(t_{0} \mid t_{0}\right)$ is a $(L \times L)$ diagonal matrix, contains initial error covariance for matrix $\widetilde{\mathbf{K}}$. In the present study, a value of 1.0 is considered for the diagonal entries of $\mathbf{P}_{x}\left(t_{0} \mid t_{0}\right)$. Hoshiya and Saito [42] and Jazwinski [30] pointed out that the diagonal entries for $\mathbf{P}_{k}\left(t_{0} \mid t_{0}\right)$ should be large positive numbers to accelerate the convergence of the local iteration process. A value of 1000 is used in this study.

The basic filtering process in EKF is the same as Kalman filter (KF), that is, propagation of the state mean and covariance from time $t_{k}$ to one step forward in time $t_{k+1}$, and then updating them when the measurement at time $t_{k+1}$ becomes available. Mathematically, the steps can be expressed as.

(i) Prediction of state mean $\hat{\mathbf{Z}}\left(t_{k+1} \mid t_{k}\right)$ and its error covariance matrix $\mathbf{P}\left(t_{k+1} \quad \mid t_{k}\right)$ for the next time increment $t_{k+1}$ as:

$$
\begin{aligned}
\hat{\mathbf{Z}}\left(t_{k+1} \mid t_{k}\right)= & \hat{\mathbf{Z}}\left(t_{k} \mid t_{k}\right)+\int_{t_{k}}^{t_{k+1}} \hat{\mathbf{Z}}\left(t \mid t_{k}\right) d t, \\
\mathbf{P}\left(t_{k+1} \mid t_{k}\right)= & \boldsymbol{\Phi}\left[t_{k+1}, t_{k} ; \hat{\mathbf{Z}}\left(t_{k} \mid t_{k}\right)\right] \cdot \mathbf{P}\left(t_{k} \mid t_{k}\right) \\
& \bullet \Phi^{\mathrm{T}}\left[t_{k+1}, t_{k} ; \hat{\mathbf{Z}}\left(t_{k} \mid t_{k}\right)\right] .
\end{aligned}
$$

(ii) Using measurement $\mathbf{Y}\left(t_{k+1} \mid t_{k}\right)$ and Kalman gain $\mathbf{K}\left[t_{k+1} ; \hat{\mathbf{Z}}\left(t_{k+1} \mid t_{k}\right)\right]$ available at time $t_{k+1}$, updated state mean $\hat{\mathbf{Z}}\left(t_{k+1} \mid t_{k+1}\right)$ and error covariance matrix $\mathbf{P}\left(t_{k+1} \mid t_{k+1}\right)$ can be obtained as

$$
\begin{aligned}
\hat{\mathbf{Z}}\left(t_{k+1} \mid t_{k+1}\right) & \\
= & \hat{\mathbf{Z}}\left(t_{k+1} \mid t_{k}\right)+\mathbf{K}\left[t_{k+1} ; \hat{\mathbf{Z}}\left(t_{k+1} \mid t_{k}\right)\right] \\
& \bullet\left\{\mathbf{Y}\left(t_{k+1}\right)-h\left[\hat{\mathbf{Z}}\left(t_{k+1} \mid t_{k}\right), t_{k+1}\right]\right\},
\end{aligned}
$$

$$
\begin{aligned}
& \mathbf{P}\left(t_{k+1} \mid t_{k+1}\right) \\
& =\left\{\mathbf{I}-\mathbf{K}\left[t_{k+1} ; \hat{\mathbf{Z}}\left(t_{k+1} \mid t_{k}\right)\right] \bullet \mathbf{M}\left[t_{k+1} ; \hat{\mathbf{Z}}\left(t_{k+1} \mid t_{k}\right)\right]\right\} \\
& \bullet \mathbf{P}\left(t_{k+1} \mid t_{k}\right) \bullet\left\{\mathbf{I}-\mathbf{K}\left[t_{k+1} ; \hat{\mathbf{Z}}\left(t_{k+1} \mid t_{k}\right)\right]\right. \\
& \left.\bullet \mathbf{M}\left[t_{k+1} ; \hat{\mathbf{Z}}\left(t_{k+1} \mid t_{k}\right)\right]\right\}^{T} \\
& +\mathbf{K}\left[t_{k+1} ; \hat{\mathbf{Z}}\left(t_{k+1} \mid t_{k}\right)\right] \bullet \mathbf{R}\left(t_{k+1}\right) \bullet \mathbf{K}^{\mathrm{T}}\left[t_{k+1} ; \hat{\mathbf{Z}}\left(t_{k+1} \mid t_{k}\right)\right],
\end{aligned}
$$

where $\Phi\left[t_{k+1}, t_{k} ; \hat{\mathbf{Z}}\left(t_{k} \mid t_{k}\right)\right]$ is the state transfer matrix from $t_{k}$ to $t_{k+1} ; \mathbf{K}\left[t_{k+1} ; \hat{\mathbf{Z}}\left(t_{k+1} \mid t_{k}\right)\right]$ and $\mathbf{R}\left(t_{k+1}\right)$ is the Kalman gain matrix and diagonal noise covariance matrix, respectively, at time $t_{k+1}$. Detail procedure for calculation of $\boldsymbol{\Phi}, \mathbf{K}$, and $\mathbf{M}$ can be found in [58]. The symbol • stands for matrix multiplication. In the present study, diagonal entries in the noise covariance matrix $\mathbf{R}_{t k}$ are considered to be $10^{-4}$.

Prediction and updating processes are successively carried out for each of the $m$ time points for the entire time history used for the identification. As mentioned earlier, a weight factor $w$ is introduced after completion of the first global iteration process. To start the second global iteration process, $\hat{\mathbf{Z}}^{(1)}\left(t_{m} \mid t_{m}\right)$ and $\mathbf{P}^{(1)}\left(t_{m} \mid t_{m}\right)$, obtained at the end of first global iteration process, are used and a weight factor $w$ (a large positive number) is introduced into the error covariance matrix to amplify the covariance values of the stiffness parameters. In this study, $w$ is considered to be 10 and 1000 depending on the applications. The same prediction and updating processes of local iterations are carried out for all the time points, and a new set of state vector and error covariance matrix are obtained at the end of second global iteration. The global iteration processes are continued until the estimated error of identified stiffness parameters at the end of two consecutive global iterations becomes smaller than a predetermined convergence criterion $(\varepsilon)$. Considering the magnitude of the stiffness parameter of the elements for the frame shown in Figure $1, \varepsilon$ considered to be $10 \mathrm{kN}-\mathrm{m}$ in this study. If they diverge, the best estimated values are considered based on minimum objective function $\bar{\theta}$ as suggested by Hoshiya and Saito [42].

\section{Example: Health Assessment of a Three-Bay Three-Story Frame}

Some of the recent advances in the GILS-EKF-UI procedure to assess defect-free and defective states of a structure at the element level considering several issues discussed earlier are illustrated with the help of a relatively large structural system, as discussed next.

5.1. Description of the Frame. A two-dimensional frame with a bay width of $9.14 \mathrm{~m}$ and story height of $3.66 \mathrm{~m}$, as shown in Figure 1, is considered. The frame has a total of 21 members: 9 beams and 12 columns. The beams and columns are made of W21 $\times 68$ and W14 $\times 61$ sections, respectively, of Grade 50 steel. The frame is modeled by 
16 nodes in the FE representation. Each node has three dynamic degrees of freedom (DDOFs): two translational and one rotational. The support condition at the base (nodes $13,14,15$, and 16) of the frame is considered to be fixed. The total number of DDOFs for the frame is 36 . The actual theoretical stiffness parameter values $k_{i}$ evaluated in terms of $\left(E_{i} I_{i} / L_{i}\right)$ are calculated to be $13476 \mathrm{kN}-\mathrm{m}$ and $14553 \mathrm{kN}$ $\mathrm{m}$ for a typical beam and a column, respectively. First two natural frequencies of the frame are estimated to be $f_{1}=$ $6.0023 \mathrm{~Hz}$ and $f_{2}=18.944 \mathrm{~Hz}$, respectively. Following the procedure described in Clough and Penzien [66], Rayleigh damping coefficient $\alpha$ and $\beta$ are calculated to be 2.86393 and 0.00063799 , respectively, for an equivalent modal damping of $5 \%$ (commonly used in model codes in the US) of the critical for the first two modes.

To demonstrate the robustness of the GILS-EKF-UI method, the frame is excited simultaneously by two impulsive loadings applied at node 1 and 9 in the horizontal direction, as shown in Figure 1, instead of one. Each load has a magnitude of $45 \mathrm{kN}$ and acts for a duration of $0.05 \mathrm{~s}$. Instead of conducting the experiments and following the general practices, the information on responses are analytically generated using a commercially available software ANSYS (ver. 11) [67]. The responses are obtained at $0.00025 \mathrm{~s}$ time interval. After the responses are simulated, the information on input excitations is completely ignored. Responses between $0.05 \mathrm{~s}$ and $0.37 \mathrm{~s}$ providing 1281 time points are used in the subsequent health assessment process.

5.2. Health Assessment of Defect-Free Frame. The defectfree state of the frame is considered first. The substructure required in Stage 1 to implement the procedure is shown in Figure 1. Essentially, to identify the whole frame, total number of responses measured at all 15 DDOFs (responses at nodes 1, 2, 5, 9, and 10) of the substructure are required and are assumed to be available. Following the procedures to implement Stage 1, the stiffness parameters of the five elements in the substructure and the excitation time histories are identified. The theoretical and identified stiffness parameters are summarized in Table 1(a). The differences between the theoretical and identified stiffness parameters, generally denoted in the literature as the error in the identification expressed in percentage, are then estimated. The maximum error in the stiffness identification is $0.094 \%$. About $10 \%$ error in the identification was reported to be acceptable even when excitation information was used in the identification process $[42,43,46]$.

With the information on identified stiffness parameters and unknown input excitations, Stage 2 is initiated to identify and to assess the health of the whole frame. The issues related to the absolute minimum numbers of required responses, the corresponding errors in the identification, and the optimum or ideal numbers are still open questions in the literature. To study the issue comprehensively, the identified stiffness parameters for the whole frame using responses at 15 DDOFs (nodes 1, 2, 5, 9, and 10), 18 DDOFs (nodes 1, 2, 3, 5, 9, and 10), 21 DDOFs (nodes 1, 2, 3, 4, 5, 9, and 10), and 24 DDOFs (nodes 1, 2, 3, 4, 5, 9, 10, and 12) are summarized in columns $3,4,5$, and 6 , respectively, in Table $1(\mathrm{~b})$. As expected, the accuracy in the identification increases as more responses are used. In fact, if all response information is used, the accuracy in the identification is expected to be similar to that of the substructure. The errors in identification using responses at 15,18, and 21 DDOFs (not shown in the table) vary significantly for this example. The errors in the identification are expected to be dependent on many factors including the total duration of responses used for the health assessment, the time interval of the measurements, errors in the state vector and the measurement noises represented by $\mathbf{R}_{t k}$, the weight factor $(w)$, etc. It is difficult to make any general statement on errors in identification, since it is problem dependent. The values of $\mathbf{R}_{t k}$ and $w$ are summarized in the table. For the assumed conditions and the problem under consideration, the maximum error in the identification for 24 DDOFs is found to be $4.29 \%$ and is considered to be optimal. All subsequent discussions will be made using this as the reference case. From the results summarized in Table 1 (b), it can be observed that the errors in identification do not vary significantly from members to members indicating the frame is defect-free. This example also demonstrates the benefit of extra response information.

5.3. Health Assessment of Defective Frame. One of the major reasons of NDIs of structures is to locate defect, if any, and its severity. As mentioned earlier, many types of defects are expected in structures and multiple structural elements can have defects at the time of inspection. Furthermore, after identifying a defective member, it will also be very desirable if the defect location can be identified within the defective member more accurately. This will be very attractive, particularly, when the length of the defective member is relatively long. Limiting inspection within a smaller segment of the defective member will reduce the interruption of the normal use of the structure. The GILSEKF-UI method can be used for all these purposes, as discussed in the following sections.

5.3.1. Defect 1: Reduction of Stiffness of a Single Member
by Different Magnitudes. In defect case 1, member 15 connecting nodes 9 and 10, is considered to be defective. The moment of inertia of the member over the entire length is considered to be reduced by different magnitudes, that is, $15 \%, 30 \%, 50 \%$, and $90 \%$ of the defect-free value, to study the detection capability of the method to indicate severity of defect. Obviously, the location and the severity of defects will be unknown at the time of inspection, but the measured responses should reflect their presence when the initial defect-free FE representation of the frame is used. As mentioned earlier, for this illustrative example, the responses are analytically generated using ANSYS by appropriately modeling the location, nature, and extent of defects. In real inspections, they will be measured.

The substructure required in Stage 1 is considered to be the same as used previously. It is to be noted that in this case, the substructure contains the defective element. Using responses at 15 DDOFs, the substructure is identified for different levels of degradation and the results are summarized 
TABLE 1: Stiffness parameter (EI/L) identification for defect-free frame using GILS-EKF-UI.

(a) Stage 1: Identification of substructure

\begin{tabular}{lccc}
\hline \multirow{2}{*}{ Member } & \multicolumn{3}{c}{ Identified $(E I / L)$ values in $(\mathrm{kN}-\mathrm{m})$} \\
& Nominal/Theoretical & Identified & Error $(\%)$ \\
\hline$(1)$ & $12)$ & $(3)$ & $(4)$ \\
\hline$k_{1}$ & 13476 & 13479 & 0.024 \\
$k_{4}$ & 14553 & 14556 & 0.021 \\
$k_{11}$ & 14553 & 14567 & 0.093 \\
$k_{15}$ & 13476 & 13489 & 0.094 \\
$k_{18}$ & 14553 & 14567 & 0.093 \\
\hline
\end{tabular}

(b) Stage 2: Identification of whole frame

\begin{tabular}{|c|c|c|c|c|c|c|}
\hline \multirow{3}{*}{ Member } & \multirow{3}{*}{ Nominal/Theoretical } & \multicolumn{5}{|c|}{ Identified $(E I / L)$ values in $(\mathrm{kN}-\mathrm{m})$ for the whole frame } \\
\hline & & \multirow{2}{*}{$\begin{array}{l}15 \text { DDOFs } \\
\text { Identified }\end{array}$} & \multirow{2}{*}{$\begin{array}{l}18 \text { DDOFs } \\
\text { Identified }\end{array}$} & \multirow{2}{*}{$\begin{array}{c}21 \text { DDOFs } \\
\text { Identified }\end{array}$} & \multicolumn{2}{|c|}{24 DDOFs } \\
\hline & & & & & Identified & Error $(\%)$ \\
\hline$(1)$ & $(2)$ & (3) & $(4)$ & $(5)$ & $(6)$ & $(7)$ \\
\hline$k_{1}$ & 13476 & 11549 & 14249 & 13473 & 13545 & 0.51 \\
\hline$k_{2}$ & 13476 & 6384 & 15241 & 13244 & 13681 & 1.52 \\
\hline$k_{3}$ & 13476 & 24229 & 8321 & 13779 & 13497 & 0.16 \\
\hline$k_{8}$ & 13476 & 2930 & 10149 & 11680 & 13109 & -2.73 \\
\hline$k_{9}$ & 13476 & 50270 & 15249 & 11170 & 13519 & 0.32 \\
\hline$k_{10}$ & 13476 & 40685 & 17062 & 16639 & 13458 & -0.14 \\
\hline$k_{15}$ & 13476 & 12047 & 10015 & 11737 & 13153 & -2.40 \\
\hline$k_{16}$ & 13476 & 6082 & 10387 & 10303 & 13705 & 1.70 \\
\hline$k_{17}$ & 13476 & 14577 & 17322 & 18879 & 13808 & 2.46 \\
\hline$k_{4}$ & 14553 & 27805 & 16037 & 14514 & 14601 & 0.33 \\
\hline$k_{5}$ & 14553 & 10595 & 15485 & 14410 & 14542 & -0.08 \\
\hline$k_{6}$ & 14553 & 11857 & 12273 & 14693 & 14547 & -0.04 \\
\hline$k_{7}$ & 14553 & 13325 & 24412 & 14333 & 14432 & -0.83 \\
\hline$k_{11}$ & 14553 & 11798 & 10859 & 12672 & 14192 & -2.48 \\
\hline$k_{12}$ & 14553 & 9602 & 10497 & 11716 & 14552 & -0.01 \\
\hline$k_{13}$ & 14553 & 14115 & 18201 & 14494 & 14903 & 2.41 \\
\hline$k_{14}$ & 14553 & 22920 & 21218 & 21784 & 14547 & -0.04 \\
\hline$k_{18}$ & 14553 & 17465 & 10272 & 12572 & 14375 & -1.22 \\
\hline$k_{19}$ & 14553 & 10073 & 11568 & 11671 & 14312 & -1.66 \\
\hline$k_{20}$ & 14553 & 9499 & 19770 & 13730 & 14351 & -1.39 \\
\hline$k_{21}$ & 14553 & 28112 & 17496 & 20806 & 15177 & 4.29 \\
\hline $\mathbf{R}_{t k}$ & \multicolumn{6}{|c|}{$10^{-4}$} \\
\hline$w$ & & 1000 & 1000 & 1000 & & \\
\hline
\end{tabular}

in Table 2(a). Then, using responses at $24 \mathrm{DDOFs}$ (nodes 1 , $2,3,4,5,9,10$, and 12), the whole frame is identified. The results are presented in Table 2(b). The maximum reduction of the stiffness parameter for member 15 is found to be $15.4 \%, 28.7 \%, 47.4 \%$, and $88.9 \%$ for the four different defect scenarios. For the health assessment purpose, the changes in the stiffness parameters are critical and not their identified values. In all 4 cases, the maximum changes occur in member 15 , indicating it contains the defect and the GILS-EKF-UI method correctly identified the location and the severity of the defects. The weight factor $(w)$ used in Stage 2 are indicated at the bottom of Table 2(b).
5.3.2. Defect 2: Reduction of Stiffness of Multiple Members by Different Magnitudes. In defect case 2, two members at a time are considered to be defective. Three different combinations are considered: (a) the stiffness parameter of members 1 and 3 are reduced by $30 \%$ and $20 \%$, respectively, (b) the stiffness parameter of members 15 and 17 are reduced by $30 \%$ and $20 \%$, respectively, and (c) the stiffness parameter of members 1 and 17 are reduced by $30 \%$ and $20 \%$, respectively. It is important to note that in all these cases, one defective member is not in the substructure. Following the similar procedures as discussed for defect case 1 , the structural responses are first analytically generated 
TABLE 2: Stiffness parameter (EI/L) identification for defect 1 using GILS-EKF-UI.

(a) Stage 1: Identification of substructure

\begin{tabular}{|c|c|c|c|c|c|c|c|c|c|}
\hline \multirow{3}{*}{ Member } & \multirow{3}{*}{ Nominal/Theoretical } & \multicolumn{8}{|c|}{ Identified $(E I / L)$ values in $(\mathrm{kN}-\mathrm{m})$ for the substructure } \\
\hline & & \multicolumn{2}{|c|}{$k_{15}$ reduced by $15 \%$} & \multicolumn{2}{|c|}{$k_{15}$ reduced by $30 \%$} & \multicolumn{2}{|c|}{$k_{15}$ reduced by $50 \%$} & \multicolumn{2}{|c|}{$k_{15}$ reduced by $90 \%$} \\
\hline & & Identified & Error $(\%)$ & Identified & Error $(\%)$ & Identified & Error $(\%)$ & Identified & Error $(\%)$ \\
\hline$(1)$ & $(2)$ & (3) & $(4)$ & $(5)$ & $(6)$ & $(7)$ & $(8)$ & $(9)$ & $(10)$ \\
\hline$k_{1}$ & 13476 & 13479 & 0.02 & 13479 & 0.02 & 13479 & 0.02 & 13479 & 0.02 \\
\hline$k_{4}$ & 14553 & 14556 & 0.02 & 14556 & 0.02 & 14556 & 0.02 & 14556 & 0.02 \\
\hline$k_{11}$ & 14553 & 14580 & 0.19 & 14594 & 0.28 & 14613 & 0.41 & 14653 & 0.69 \\
\hline$k_{15}$ & 13476 & 11476 & -14.8 & 9460 & -29.8 & 6766 & -49.7 & 1357 & -89.9 \\
\hline$k_{18}$ & 14553 & 14580 & 0.19 & 14594 & 0.28 & 14614 & 0.42 & 14655 & 0.70 \\
\hline
\end{tabular}

(b) Stage 2: Identification of whole frame

\begin{tabular}{|c|c|c|c|c|c|c|c|c|c|}
\hline \multirow{4}{*}{ Member } & \multirow{4}{*}{ Nominal/Theoretical } & \multicolumn{8}{|c|}{ Identified $(E I / L)$ values in $(\mathrm{kN}-\mathrm{m})$ for the whole frame } \\
\hline & & \multirow{2}{*}{\multicolumn{2}{|c|}{$\begin{array}{l}k_{15} \text { reduced by } 15 \% \\
24 \text { DDOFs }\end{array}$}} & \multirow{2}{*}{\multicolumn{2}{|c|}{$\begin{array}{c}k_{15} \text { reduced by } 30 \% \\
24 \text { DDOFs }\end{array}$}} & \multirow{2}{*}{\multicolumn{2}{|c|}{$\begin{array}{c}k_{15} \text { reduced by } 50 \% \\
24 \text { DDOFs }\end{array}$}} & \multirow{2}{*}{\multicolumn{2}{|c|}{$\begin{array}{c}k_{15} \text { reduced by } 90 \% \\
24 \text { DDOFs }\end{array}$}} \\
\hline & & & & & & & & & \\
\hline & & Identified & Error $(\%)$ & Identified & Error $(\%)$ & Identified & Error $(\%)$ & Identified & Error $(\%)$ \\
\hline$(1)$ & $(2)$ & $(3)$ & $(4)$ & $(5)$ & $(6)$ & $(7)$ & $(8)$ & $(9)$ & $(10)$ \\
\hline$k_{1}$ & 13476 & 13660 & 1.4 & 13800 & 2.4 & 14022 & 4.0 & 14132 & 4.9 \\
\hline$k_{2}$ & 13476 & 13660 & 1.4 & 13604 & 0.9 & 13516 & 0.3 & 14011 & 4.0 \\
\hline$k_{3}$ & 13476 & 13403 & -0.5 & 13339 & -1.0 & 13224 & -1.9 & 12015 & -10.8 \\
\hline$k_{8}$ & 13476 & 13309 & -1.2 & 13545 & 0.5 & 13884 & 3.0 & 13338 & -1.0 \\
\hline$k_{9}$ & 13476 & 13124 & -2.6 & 12722 & -5.6 & 12037 & -10.7 & 12318 & -8.6 \\
\hline$k_{10}$ & 13476 & 13612 & 1.0 & 13796 & 2.4 & 14257 & 5.8 & 15219 & 12.9 \\
\hline$k_{15}$ & 13476 & 11407 & -15.4 & 9610 & -28.7 & 7085 & -47.4 & 1496 & -88.9 \\
\hline$k_{16}$ & 13476 & 13343 & -1.0 & 12960 & -3.8 & 12372 & -8.2 & 11843 & -12.1 \\
\hline$k_{17}$ & 13476 & 13453 & -0.2 & 13197 & -2.1 & 12958 & -3.8 & 10902 & -19.1 \\
\hline$k_{4}$ & 14553 & 14850 & 2.0 & 15135 & 4.0 & 15635 & 7.4 & 16068 & 10.4 \\
\hline$k_{5}$ & 14553 & 14612 & 0.4 & 14657 & 0.7 & 14757 & 1.4 & 15803 & 8.6 \\
\hline$k_{6}$ & 14553 & 14471 & -0.6 & 14366 & -1.3 & 14096 & -3.1 & 13402 & -7.9 \\
\hline$k_{7}$ & 14553 & 14184 & -2.5 & 13952 & -4.1 & 13491 & -7.3 & 12110 & -16.8 \\
\hline$k_{11}$ & 14553 & 14558 & 0.0 & 14985 & 3.0 & 15573 & 7.0 & 14642 & 0.6 \\
\hline$k_{12}$ & 14553 & 14706 & 1.1 & 14796 & 1.7 & 14743 & 1.3 & 14370 & -1.3 \\
\hline$k_{13}$ & 14553 & 14929 & 2.6 & 14924 & 2.5 & 15081 & 3.6 & 18502 & 27.1 \\
\hline$k_{14}$ & 14553 & 14292 & -1.8 & 14098 & -3.1 & 13938 & -4.2 & 13103 & -10.0 \\
\hline$k_{18}$ & 14553 & 14682 & 0.9 & 15029 & 3.3 & 15415 & 5.9 & 14668 & 0.8 \\
\hline$k_{19}$ & 14553 & 14417 & -0.9 & 14477 & -0.5 & 14466 & -0.6 & 15039 & 3.3 \\
\hline$k_{20}$ & 14553 & 14651 & 0.7 & 14847 & 2.0 & 15158 & 4.2 & 18497 & 27.1 \\
\hline$k_{21}$ & 14553 & 14905 & 2.4 & 14745 & 1.3 & 14660 & 0.7 & 12948 & -11.0 \\
\hline $\mathbf{R}_{t k}$ & \multicolumn{9}{|c|}{$10^{-4}$} \\
\hline$w$ & & \multicolumn{2}{|c|}{1000} & \multicolumn{2}{|c|}{1000} & \multicolumn{2}{|c|}{1000} & \multicolumn{2}{|c|}{10} \\
\hline
\end{tabular}

in the presence of defects. Using the same substructure as shown in Figure 1, and using responses at 15 DDOFs, it is identified. The results are summarized in Table 3(a). Then, using responses at $24 \mathrm{DDOF}$, as in defect case 1 , the whole frame is identified and the results are presented in Table 3(b).

The results clearly indicate the locations of the defective members and the severity of defects in them. This example establishes that the GILS-EKF-UI method can identify different defective members and different levels of degradation in them and the defective member need not be in the substructure. The weight factor $(w)$ used in this work are indicated at the bottom of Table 3(b).

5.3.3. Defect 3: Locating Less Severe Defect-Loss of Area over a Finite Length of a Member. After successfully identifying different magnitudes of defects in single and multiple members, the capability of the procedure is checked if it can identify less severe defect. The cross-sectional area of member 16, a beam at the first story level, is considered to be corroded over a length of $40 \mathrm{~cm}$, located at a distance 
TABLe 3: Stiffness parameter (EI/L) identification for defect 2 using GILS-EKF-UI.

(a) Stage 1: Identification of substructure

\begin{tabular}{|c|c|c|c|c|c|c|c|}
\hline \multirow{3}{*}{ Member } & \multirow{3}{*}{ Nominal/Theoretical } & \multicolumn{6}{|c|}{ Identified $(E I / L)$ values in $(\mathrm{kN}-\mathrm{m})$ for the substructure } \\
\hline & & \multicolumn{2}{|c|}{$\begin{array}{l}k_{1}, k_{3} \text { reduced by } \\
30 \%, 20 \% \text {, resp. }\end{array}$} & \multicolumn{2}{|c|}{$\begin{array}{c}k_{15}, k_{17} \text { reduced by } \\
30 \%, 20 \% \text {, resp. }\end{array}$} & \multicolumn{2}{|c|}{$\begin{array}{c}k_{1}, k_{17} \text { reduced by } \\
30 \%, 20 \% \text {, resp. }\end{array}$} \\
\hline & & Identified & Error $(\%)$ & Identified & Error $(\%)$ & Identified & Error $(\%)$ \\
\hline (1) & (2) & (3) & (4) & (5) & (6) & (7) & (8) \\
\hline$k_{1}$ & 13476 & 9451 & -29.87 & 13479 & 0.02 & 9451 & -29.87 \\
\hline$k_{4}$ & 14553 & 14578 & 0.17 & 14556 & 0.02 & 14579 & 0.18 \\
\hline$k_{11}$ & 14553 & 14567 & 0.10 & 14593 & 0.28 & 14566 & 0.09 \\
\hline$k_{15}$ & 13476 & 13489 & 0.10 & 9459 & -29.81 & 13489 & 0.09 \\
\hline$k_{18}$ & 14553 & 14567 & 0.10 & 14594 & 0.28 & 14567 & 0.09 \\
\hline
\end{tabular}

(b) Stage 2: Identification of whole frame

\begin{tabular}{|c|c|c|c|c|c|c|c|}
\hline \multirow{5}{*}{ Member } & \multirow{5}{*}{ Nominal/Theoretical } & \multicolumn{6}{|c|}{ Identified $(E I / L)$ values in $(\mathrm{kN}-\mathrm{m})$ for the whole frame } \\
\hline & & \multirow{3}{*}{\multicolumn{2}{|c|}{$\begin{array}{c}k_{1}, k_{3} \text { reduced by } \\
30 \%, 20 \% \text {, resp. } \\
24 \text { DDOFs }\end{array}$}} & \multirow{3}{*}{\multicolumn{2}{|c|}{$\begin{array}{c}k_{15}, k_{17} \text { reduced by } \\
30 \%, 20 \% \text {, resp. } \\
24 \text { DDOFs }\end{array}$}} & \multirow{3}{*}{\multicolumn{2}{|c|}{$\begin{array}{c}k_{1}, k_{17} \text { reduced by } \\
30 \%, 20 \% \text {, resp. } \\
24 \text { DDOFs }\end{array}$}} \\
\hline & & & & & & & \\
\hline & & & & & & & \\
\hline & & Identified & Error (\%) & Identified & Error (\%) & Identified & Error (\%) \\
\hline (1) & (2) & (3) & $(4)$ & $(5)$ & $(6)$ & $(7)$ & (8) \\
\hline$k_{1}$ & 13476 & 9614 & -28.7 & 13768 & 2.2 & 9527 & -29.3 \\
\hline$k_{2}$ & 13476 & 13101 & -2.8 & 13629 & 1.1 & 12865 & -4.5 \\
\hline$k_{3}$ & 13476 & 11283 & -16.3 & 13403 & -0.5 & 13209 & -2.0 \\
\hline$k_{8}$ & 13476 & 13402 & -0.6 & 13180 & -2.2 & 12142 & -9.9 \\
\hline$k_{9}$ & 13476 & 12894 & -4.3 & 12647 & -6.2 & 13425 & -0.4 \\
\hline$k_{10}$ & 13476 & 13110 & -2.7 & 13912 & 3.2 & 14086 & 4.5 \\
\hline$k_{15}$ & 13476 & 13433 & -0.3 & 9352 & -30.6 & 12539 & -7.0 \\
\hline$k_{16}$ & 13476 & 13427 & -0.4 & 13519 & 0.3 & 14085 & 4.5 \\
\hline$k_{17}$ & 13476 & 14533 & 7.8 & 10729 & -20.4 & 11095 & -17.7 \\
\hline$k_{4}$ & 14553 & 14485 & -0.5 & 15012 & 3.2 & 14752 & 1.4 \\
\hline$k_{5}$ & 14553 & 13924 & -4.3 & 14606 & 0.4 & 14754 & 1.4 \\
\hline$k_{6}$ & 14553 & 14830 & 1.9 & 14391 & -1.1 & 14458 & -0.7 \\
\hline$k_{7}$ & 14553 & 15568 & 7.0 & 14034 & -3.6 & 14056 & -3.4 \\
\hline$k_{11}$ & 14553 & 14616 & 0.4 & 14667 & 0.8 & 13472 & -7.4 \\
\hline$k_{12}$ & 14553 & 14583 & 0.2 & 14833 & 1.9 & 14378 & -1.2 \\
\hline$k_{13}$ & 14553 & 13795 & -5.2 & 15026 & 3.3 & 15606 & 7.2 \\
\hline$k_{14}$ & 14553 & 15458 & 6.2 & 14224 & -2.3 & 14996 & 3.0 \\
\hline$k_{18}$ & 14553 & 14668 & 0.8 & 14887 & 2.3 & 14010 & -3.7 \\
\hline$k_{19}$ & 14553 & 14109 & -3.1 & 14601 & 0.3 & 14354 & -1.4 \\
\hline$k_{20}$ & 14553 & 13443 & -7.6 & 14608 & 0.4 & 14700 & 1.0 \\
\hline$k_{21}$ & 14553 & 15719 & 8.0 & 14811 & 1.8 & 14955 & 2.8 \\
\hline $\mathbf{R}_{t k}$ & \multicolumn{7}{|c|}{$10^{-4}$} \\
\hline$w$ & & \multicolumn{2}{|c|}{1000} & \multicolumn{2}{|c|}{1000} & \multicolumn{2}{|c|}{10} \\
\hline
\end{tabular}

of $1.2 \mathrm{~m}$ from node 10, as shown in Figure 2. The web and flange thicknesses are considered to be reduced to one fourth of their original values. The loss of thicknesses will result in the reduction of the cross-sectional area by $75.23 \%$ and the moment of inertia by $76.40 \%$ from the nominal values. To simulate measured responses analytically, in the FE representation, a new element with the reduced crosssectional properties is introduced. The defective frame is then excited by the impulsive loadings, and the responses are calculated using ANSYS. Then, as before, after identifying the substructure, the whole frame is identified using responses at 24 DDOFs. The identified stiffness parameters are shown in column 3 of Table 4(a) the substructure, and column 3 of Table 4(b) for the whole structure. The stiffness parameter for member 16 is reduced by the maximum amount of $13.8 \%$, significantly more than other members, indicating that it is defective. It can be concluded that the GILS-EKF-UI method successfully located the less severe defective member. 


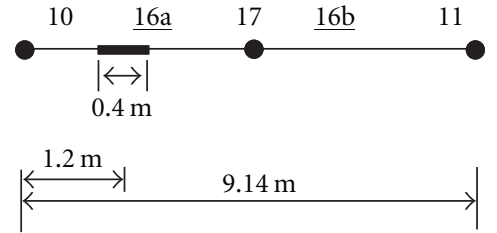

Figure 2: Identification of less severe defect more accurately.

5.4. Locating Defect More Accurately in a Defective Member. Beam 16 containing defect 3 in the preceding example is $9.14 \mathrm{~m}$ long. It will be desirable to locate the defective spot more accurately within the defective element. Two alternatives are explored as discussed next.

5.4.1. Alternative 1. One way to meet this objective will be to represent the defective element by a greater number of finite elements and then identify which element is defective. For the sake of discussion, member 16 is represented by two elements by introducing a new node at the mid-span of the beam. In the FE representation, they are represented by member $16 \mathrm{a}$ and $16 \mathrm{~b}$, as shown in Figure 2 . Since the defective member is known, two more sensors are placed at node 11 and new node 17 for this exploratory example. The substructure is considered to be same as considered in previous cases. After identifying the substructure, the whole frame is identified using response at 30 DDOFs (nodes 1, 2, 3, $4,5,9,10,11,12$, and 17). The identified results are shown in column 5 of Table 4 (a) for the substructure and in column 6 of Table 4(b) for the whole structure. It is clear that member 16a contains the defect. Defective element 16a again can be split into two elements and the defective segment can again be similarly identified. The process can be continued until the desired length of the segment for inspection is obtained.

The new node 17 is introduced in the mid-span of the defective member $16,4.57 \mathrm{~m}$ from node 10 . It could be anywhere along the length of member 16 . To study the proximity of the new node with respect to the defect spot, the following two additional cases are considered. The location of the new node is considered to be $3.07 \mathrm{~m}$ and $2.07 \mathrm{~m}$ from node 10 . Thus, the lengths of the two elements $16 \mathrm{a}$ and $16 \mathrm{~b}$ becomes $3.07 \mathrm{~m}$ and $6.07 \mathrm{~m}$ and $2.07 \mathrm{~m}$ and $7.07 \mathrm{~m}$, respectively, for the two cases. Element 16a will contain the defect in all cases. Obviously, the defect will be much closer to node 17 in the second case than in the first case. Following the same procedures as when the new node is at the midspan, the substructures and the whole frames are identified. The identified stiffness parameters are shown in columns 9 and 12 in Table 4(b), respectively, for the two cases. In all cases, element $16 \mathrm{a}$ is correctly identified to be defective. However, when node 17 is the closest to the defect location, the defective element is identified with increased confidence. This example also demonstrates that the defective element(s) need not be in the substructure. The weight factor $(w)$ used in this work are indicated at the bottom of Table 4(b).

It is to be noted that a set of new experiments need to be conducted for this alternative, and it will cost additional money. It is presented here to document the wider implementation potential of the GILS-EKF-UI method. The total number of DDOFs used to identify the defect location may not be an issue in this alternative. Advantages of spending additional money in conducting new experiments to locate defect spot more accurately versus inspecting the whole beam need to be considered on a case by case basis. Also, Alternative 2 could be more attractive.

5.4.2. Alternative 2. Since the defective member is known, the defective spot can be identified more accurately within it by using only the GILS-UI procedure. As in Alternative 1, the defective member can be represented by two members 16 a and 16b, as shown in Figure 2. Sensors can be placed at nodes 10,11 , and 17, and the member can be excited by an impact, say of magnitude $10 \mathrm{kN}$, applied vertically downward at new node 17 . Using response at 9 DDOFs available at the three nodes, the two members are identified. The identified results are shown in column 3 of Table 5 for the two members. The identified stiffness parameter for member $16 \mathrm{a}$ is observed to be reduced much more than member $16 \mathrm{~b}$ indicating that it contains the defect.

Following the same procedure as in Alternative 1, the location of the new node is moved to the location $3.07 \mathrm{~m}$ and $2.07 \mathrm{~m}$ from node 10 . Using GILS-UI procedure, the two members are identified. The results are presented in column 6 and 9 of Table 5, respectively, for the two cases. Again, in all cases, element $16 \mathrm{a}$ is correctly identified to be defective. It can be observed that when node 17 is moved gradually close to the defect spot, the identified stiffness parameter for member 16 a shows higher reduction, and when node 17 is the closest (considering the three cases) to the defect location, the defective element is identified more easily. This exercise demonstrates that the defect spot can be located without repeating the complete structural identification using the GILS-EKF-UI procedure.

In general, Alternative 2 is expected to be more economical to implement. However, it needs to be investigated considering the location of the defective member on a case by case basis.

\section{Conclusion}

Some recent advances of a recently developed nondestructive structural health assessment procedure developed by the research team at the University of Arizona, denoted as generalized iterative least-squares extended Kalman filter with unknown input (GILS-EKF-UI), were presented in this paper. The procedure is a finite elements-based timedomain system-sidentification technique and can be used to assess health of linear or mildly nonlinear structures using only limited number of noise-contaminated response information. Measurement of input excitation(s) was not necessary. The robustness and capabilities of the method has been extended to assess health of large structural systems. With the help of examples, it is demonstrated that the structure can be excited by multiple loadings simultaneously. However, as expected, a relatively larger number of responses are required to assess health of larger structural systems. The determination of optimum number of responses required for structural identification is now under intensive investigation. 


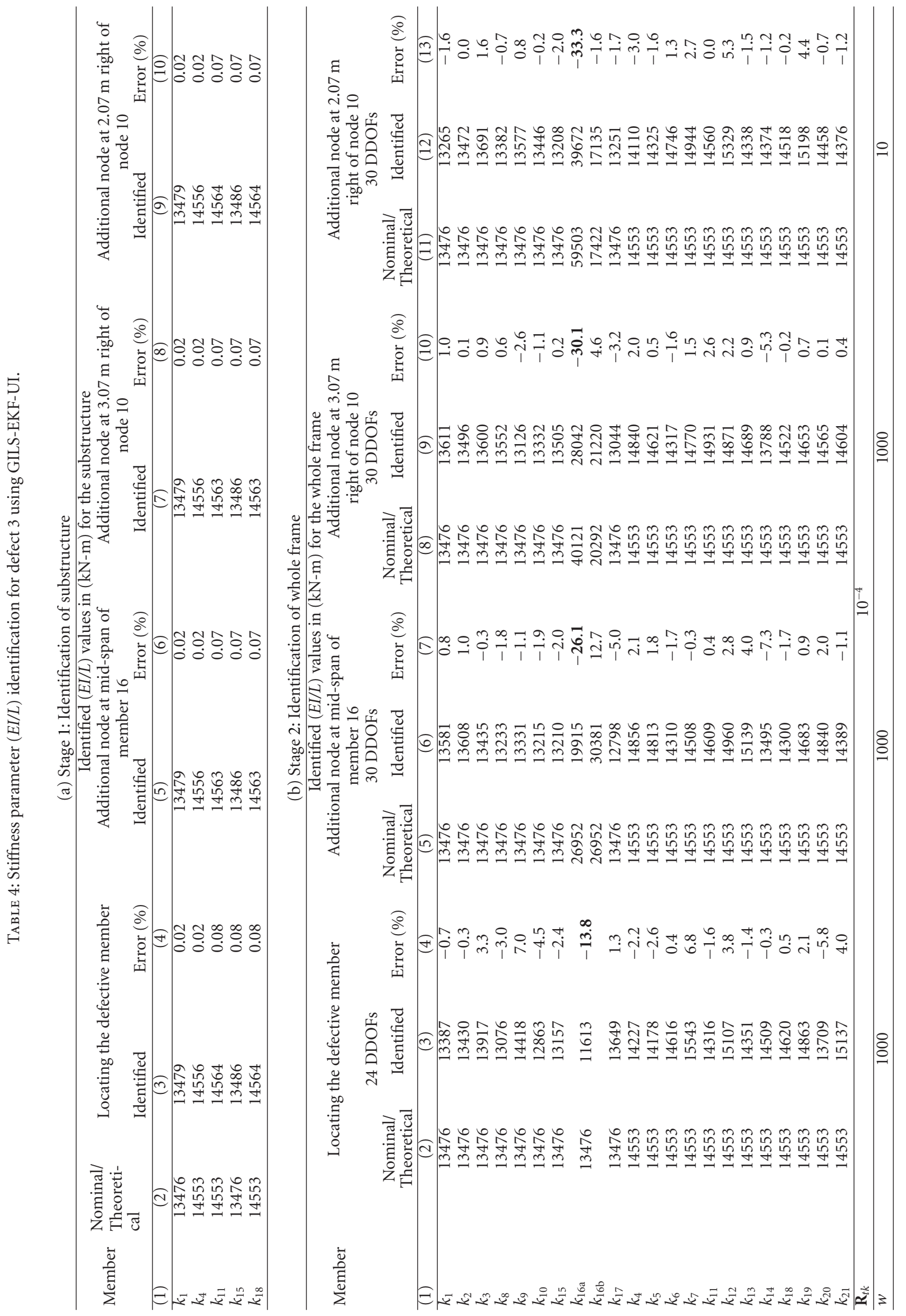


TABLE 5: Stiffness parameter (EI/L) identification for defect 3 using Stage 1 of GILS-EKF-UI.

\begin{tabular}{|c|c|c|c|c|c|c|c|c|c|}
\hline \multirow{4}{*}{ Member } & \multicolumn{9}{|c|}{ Identified $(E I / L)$ values in $(\mathrm{kN}-\mathrm{m})$ for member $16 \mathrm{a}$ and $16 \mathrm{~b}$} \\
\hline & \multirow{2}{*}{\multicolumn{3}{|c|}{$\begin{array}{l}\text { Additional node at mid-span of } \\
\text { member } 16 \\
9 \text { DDOFs }\end{array}$}} & \multirow{2}{*}{\multicolumn{3}{|c|}{$\begin{array}{c}\text { Additional node at } 3.07 \mathrm{~m} \text { right } \\
\text { of node } 10 \\
9 \text { DDOFs }\end{array}$}} & \multirow{2}{*}{\multicolumn{3}{|c|}{$\begin{array}{l}\text { Additional node at } 2.07 \mathrm{~m} \\
\text { right of node } 10 \\
9 \text { DDOFs }\end{array}$}} \\
\hline & & & & & & & & & \\
\hline & $\begin{array}{l}\text { Nominal/ } \\
\text { Theoretical }\end{array}$ & Identified & Error $(\%)$ & $\begin{array}{l}\text { Nominal/ } \\
\text { Theoretical }\end{array}$ & Identified & Error $(\%)$ & $\begin{array}{l}\text { Nominal/ } \\
\text { Theoretical }\end{array}$ & Identified & Error $(\%$ \\
\hline$(1)$ & $(2)$ & (3) & $(4)$ & $(5)$ & (6) & (7) & $(8)$ & (9) & $(10)$ \\
\hline$k_{16 \mathrm{a}}$ & 26952 & 20276 & -24.8 & 40121 & 29072 & -27.5 & 59503 & 39557 & -33.5 \\
\hline$k_{16 \mathrm{~b}}$ & 26952 & 25777 & -4.4 & 20292 & 20604 & 1.5 & 17422 & 18475 & 6.0 \\
\hline
\end{tabular}

It was observed that the method identified defects in various stages of degradation in single or multiple members and also relatively less severe defect. The defective element(s) need not necessarily be located in the substructure but the defect detection capability increases if the defect spot is closer to the substructure. Two alternatives are suggested to locate defect spot more accurately within a defective element. The paper clearly expanded the implementation potential of the GILS-EKF-UI procedure to assess health of large structural systems.

\section{References}

[1] "Redefining Global Infrastructure, Nuveen Investments," http://www. nuveen.com/Home/Documents/Viewer.aspx?file $\mathrm{Id}=51010$.

[2] "Report Card for American Infrastructure," 2005, http://apps. asce.org/reportcard/2005/index2005.cfm.

[3] "Global Infrastructure Spending to Reach \$35 Trillion Over the Next 20 Years," http://moneymorning.com/2009/02/ 05/infrastructure-stimulus-2/ .

[4] S. Saadat, M. N. Noori, G. D. Buckner, T. Furukawa, and Y. Suzuki, "Structural health monitoring and damage detection using an intelligent parameter varying (IPV) technique," International Journal of Non-Linear Mechanics, vol. 39, no. 10, pp. 1687-1697, 2004.

[5] T. V. Anh, Enhancements to the damage locating vector method for structural health monitoring, $\mathrm{Ph}$. D. dissertation, National University of Singapore, Singapore, 2009.

[6] D. Wang and A. Haldar, "An element level SI with unknown input information," Journal of Engineering Mechanics, vol. 120, no. 1, pp. 159-176, 1994.

[7] X. Ling and A. Haldar, "Element level system identification with unknown input with Rayleigh damping," Journal of Engineering Mechanics, vol. 130, no. 8, pp. 877-885, 2004.

[8] M. Katkhuda, R. Martinez-Flores, and A. Haldar, "Health assessment at local level with unknown input excitation," Journal of Structural Engineering, vol. 131, no. 6, pp. 956-965, 2005.

[9] A. Rytter, Vibration based inspection of civil engineering structures, Ph. D. dissertation, Department of Building Technology and Structural Engineering, Aalborg University, Denmark, 1993.

[10] J. S. Lew, J. N. Juang, and R. W. Longman, "Comparison of several system identification methods for flexible structures," Journal of Sound and Vibration, vol. 167, no. 3, pp. 461-480, 1993.
[11] R. Ghanem and M. Shinozuka, "Structural-system identification-I: theory," Journal of Engineering Mechanics, vol. 121, no. 2, pp. 255-264, 1995.

[12] M. Shinozuka and R. Ghanem, "Structural system identification-II: experimental verification," Journal of Engineering Mechanics, vol. 121, no. 2, pp. 265-273, 1995.

[13] S. W. Doebling, C. R. Farrar, M. B. Prime, and D. W. Shevitz, "Damage identification and health monitoring of structural and mechanical systems from changes in their vibration characteristics: a literature review," Tech. Rep. LA-13070-MS, Los Alamos National Laboratory, 1996.

[14] C. R. Farrar, S. W. Doebling et al., "A review of modalbased damage identification procedures," Tech. Rep. LA-UR97-2468, Los Alamos National Laboratory, 1997.

[15] O. S. Salawu, "Detection of structural damage through changes in frequency: a review," Engineering Structures, vol. 19, no. 9, pp. 718-723, 1997.

[16] E. P. Carden and P. Fanning, "Vibration based condition monitoring: a review," Structural Health Monitoring, vol. 3, no. 4, pp. 355-377, 2004.

[17] H. Sohn, C. R. Farrar, F. M. Hemez et al., "A review of structural health monitoring literature: 1996-2001," Tech. Rep. LA-13976-MS, Los Alamos National Laboratory, 2004.

[18] J. Humar, A. Bagchi, and H. Xu, "Performance of vibrationbased techniques for the identification of structural damage," Structural Health Monitoring, vol. 5, no. 3, pp. 215-241, 2006.

[19] G. Kerschen, K. Worden, A. F. Vakakis, and J. C. Golinval, "Past, present and future of nonlinear system identification in structural dynamics," Mechanical Systems and Signal Processing, vol. 20, no. 3, pp. 505-592, 2006.

[20] H. A. Nasrellah, Dynamic state estimation techniques for identification of parameters of finite element structural models, $\mathrm{Ph}$. D. dissertation, Indian Institute of Science, Bangalore, India, 2009.

[21] W. Fan and P. Qiao, "Vibration-based damage identification methods: a review and comparative study," Structural Health Monitoring, vol. 10, no. 1, pp. 83-111, 2011.

[22] K. Worden and J. M. Dulieu-Barton, "An overview of intelligent fault detection in systems and structures," Structural Health Monitoring, vol. 3, no. 1, pp. 85-98, 2004.

[23] C. R. Farrar and K. Worden, "An introduction to structural health monitoring," Philosophical Transactions of the Royal Society A, vol. 365, no. 1851, pp. 303-315, 2007.

[24] P. C. Chang, A. Flatau, and S. C. Liu, "Review paper: health monitoring of civil infrastructure," Structural Health Monitoring, vol. 2, no. 3, pp. 257-267, 2003.

[25] K. Worden, C. R. Farrar, G. Manson, and G. Park, "The fundamental axioms of structural health monitoring," Proceedings of the Royal Society A, vol. 463, no. 2082, pp. 1639-1664, 2007. 
[26] M. I. Friswell, "Damage identification using inverse methods," Philosophical Transactions of the Royal Society A, vol. 365, no. 1851, pp. 393-410, 2007.

[27] P. S. Maybeck, Stochastic Models, Estimation, and Control Theory, chapter 1, Academic Press, UK, 1979.

[28] R. E. Kalman, "A new approach to linear filtering and prediction problems," Transaction of the ASME, Journal of Basic Engineering, vol. 82, pp. 35-45, 1960.

[29] R. E. Kalman and R. Bucy, "New results in linear filtering and prediction theory," ASME Journal of Basic Engineering, vol. 83, pp. 95-108, 1961.

[30] A. H. Jazwinski, Stochastic Process and Filtering Theory, Academic Press, UK, 1970.

[31] G. Welch and G. Bishop, "An introduction to the Kalman filter," Tech. Rep. TR95-041, Department of Computer Science, University of North Carolina at Chapel Hill, Chapel Hill, NC, USA, 1995.

[32] G. F. Welch, "History: the use of the Kalman filter for human motion tracking in virtual reality," Presence: Teleoperators and Virtual Environments, vol. 18, no. 1, pp. 72-91, 2009.

[33] D. Simon, Optimal State Estimation: Kalman, $H_{\infty}$ and Nonlinear Approaches, John Wiley and Sons, Hoboken, NJ, USA, 2006.

[34] R. E. Kopp and R. J. Orford, "Linear regression applied to system identification for adaptive control systems," AIAA Journal, vol. 1, no. 10, pp. 2300-2306, 1963.

[35] H. Cox, "On the estimation of state variables and parameters for noisy dynamic systems," IEEE Transactions on Automatic Control, vol. 9, no. 1, pp. 5-12, 1964.

[36] C. H. Loh and Y. H. Tsaur, "Time domain estimation of structural parameters," Engineering Structures, vol. 10, no. 2, pp. 95-105, 1988.

[37] K. V. Yuen, K. I. Hoi, and K. M. Mok, "Selection of noise parameters for Kalman filter," Earthquake Engineering and Engineering Vibration, vol. 6, no. 1, pp. 49-56, 2007.

[38] R. Tipireddy, H. A. Nasrellah, and C. S. Manohar, "A Kalman filter based strategy for linear structural system identification based on multiple static and dynamic test data," Probabilistic Engineering Mechanics, vol. 24, no. 1, pp. 60-74, 2009.

[39] C. B. Yun and M. Shinozuka, "Identification of nonlinear structural dynamic systems," Journal of Structural Mechanics, vol. 8, no. 2, pp. 187-203, 1980.

[40] C. G. Koh, L. M. See, and T. Balendra, "Determination of storey stiffness of three-dimensional frame buildings," Engineering Structures, vol. 17, no. 3, pp. 179-186, 1995.

[41] A. Corigliano and S. Mariani, "Parameter identification in explicit structural dynamics: performance of the extended Kalman filter," Computer Methods in Applied Mechanics and Engineering, vol. 193, no. 36-38, pp. 3807-3835, 2004.

[42] M. Hoshiya and E. Saito, "Structural identification by extended Kalman filter," Journal of Engineering Mechanics, vol. 110, no. 12, pp. 1757-1772, 1984.

[43] K. Toki, T. Sato, and J. Kiyono, "Identification of structural parameters and input ground motion from response time histories," Structural Engineering / Earthquake Engineering, vol. 6, no. 2, pp. 413-421, 1989.

[44] L. Jeen-Shang and Z. Yigong, "Nonlinear structural identification using extended kalman filter," Computers and Structures, vol. 52, no. 4, pp. 757-764, 1994.

[45] M. Hoshiya and O. Maruyama, "Identification of running load and beam system," Journal of Engineering Mechanics, vol. 113, no. 6, pp. 813-824, 1987.

[46] Chan Ghee Koh, Lin Ming See, and Thambirajah Balendra, "Estimation of structural parameters in time domain: a substructure approach," Earthquake Engineering \& Structural Dynamics, vol. 20, no. 8, pp. 787-801, 1991.

[47] A. W. C. Oreta and T. A. Tanabe, "Localized identification of structures by Kalman filter," Structural Engineering/Earthquake Engineering, vol. 9, no. 4, pp. 217-226, 1993.

[48] A. W. C. Oreta and T. A. Tanabe, "Element identification of member properties of framed structures," Journal of Structural Engineering, vol. 120, no. 7, pp. 1961-1975, 1994.

[49] C. G. Koh and L. M. See, "Identification and uncertainty estimation of structural parameters," Journal of Engineering Mechanics, vol. 120, no. 6, pp. 1219-1236, 1994.

[50] J. N. Yang, S. Lin, H. Huang, and L. Zhou, "An adaptive extended Kalman filter for structural damage identification," Structural Control and Health Monitoring, vol. 13, no. 4, pp. 849-867, 2006.

[51] J. N. Yang, S. Pan, and H. Huang, "An adaptive extended Kalman filter for structural damage identifications II: unknown inputs," Structural Control and Health Monitoring, vol. 14, no. 3, pp. 497-521, 2007.

[52] L. Zhou, S. Wu, and J. N. Yang, "Experimental study of an adaptive extended Kalman filter for structural damage identification," Journal of Infrastructure Systems, vol. 14, no. 1, pp. 42-51, 2008.

[53] M. Hoshiya and A. Sutoh, "Kalman Filter-finite element method in identification," Journal of Engineering Mechanics, vol. 119, no. 2, pp. 197-210, 1993.

[54] S. J. Ghosh, D. Roy, and C. S. Manohar, "New forms of extended Kalman filter via transversal linearization and applications to structural system identification," Computer Methods in Applied Mechanics and Engineering, vol. 196, no. 49-52, pp. 5063-5083, 2007.

[55] X. Liu, P. J. Escamilla-Ambrosio, and N. A. J. Lieven, "Extended Kalman filtering for the detection of damage in linear mechanical structures," Journal of Sound and Vibration, vol. 325, no. 4-5, pp. 1023-1046, 2009.

[56] D. Wang and A. Haldar, "System identification with limited observations and without input," Journal of Engineering Mechanics, vol. 123, no. 5, pp. 504-510, 1997.

[57] D. Wang, Element level time domain system identification techniques with unknown input information, $\mathrm{Ph}$. D. dissertation, Department of Civil Engineering and Engineering Mechanics, University of Arizona, Tucson, Ariz, USA, 1995.

[58] K. Katkhuda and A. Haldar, "A novel health assessment technique with minimum information," Structural Control and Health Monitoring, vol. 15, no. 6, pp. 821-838, 2008.

[59] H. Katkhuda, In-service health assessment of real structures at the element level with unknown input and limited global responses, Ph. D. dissertation, Department of Civil Engineering and Engineering Mechanics, University of Arizona, Tucson, Ariz, USA, 2004.

[60] A. Haldar and A. K. Das, "Prognosis of structural health: nondestructive methods," International Journal of Performability Engineering, vol. 6, no. 5, pp. 487-498, 2010.

[61] R. Martinez-Flores, Damage assessment potential of a novel system identification technique-experimental verification, $\mathrm{Ph}$. D. dissertation, Department of Civil Engineering and Engineering Mechanics, University of Arizona, Tucson, Ariz, USA, 2005.

[62] R. Martinez-Flores and A. Haldar, "Experimental verification of a structural health assessment method without excitation information," Journal of Structural Engineering, vol. 34, no. 1, pp. 33-39, 2007. 
[63] R. Martinez-Flores, H. Katkhuda, and A. Haldar, "A novel health assessment technique with minimum information: verification," International Journal of Performability Engineering, vol. 4, no. 2, pp. 121-140, 2008.

[64] A. Haldar, R. Martinez-Flores, and H. Katkhuda, "Crack detection in existing structures using noise-contaminated dynamic responses," Theoretical and Applied Fracture Mechanics, vol. 50, no. 1, pp. 74-80, 2008.

[65] P. H. Vo and A. Haldar, "Post-processing of linear accelerometer data in structural identification," Journal of Structural Engineering, vol. 30, no. 2, pp. 123-130, 2003.

[66] R. W. Clough and J. Penzien, Dynamics of structures, McGrawHill, New York, NY, USA, 2nd edition, 1993.

[67] ANSYS version 11.0, The Engineering Solutions Company, 2007. 

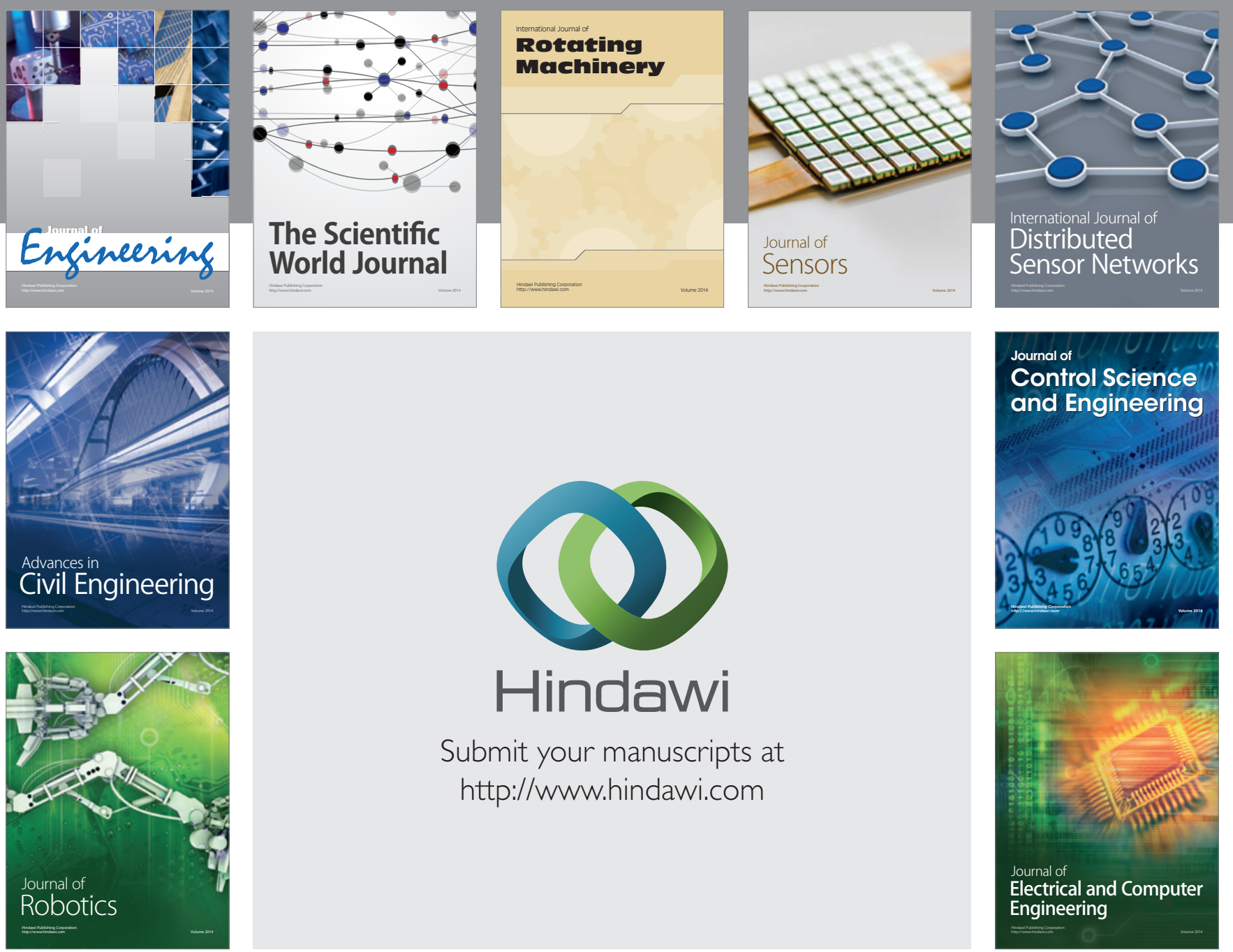

Submit your manuscripts at

http://www.hindawi.com
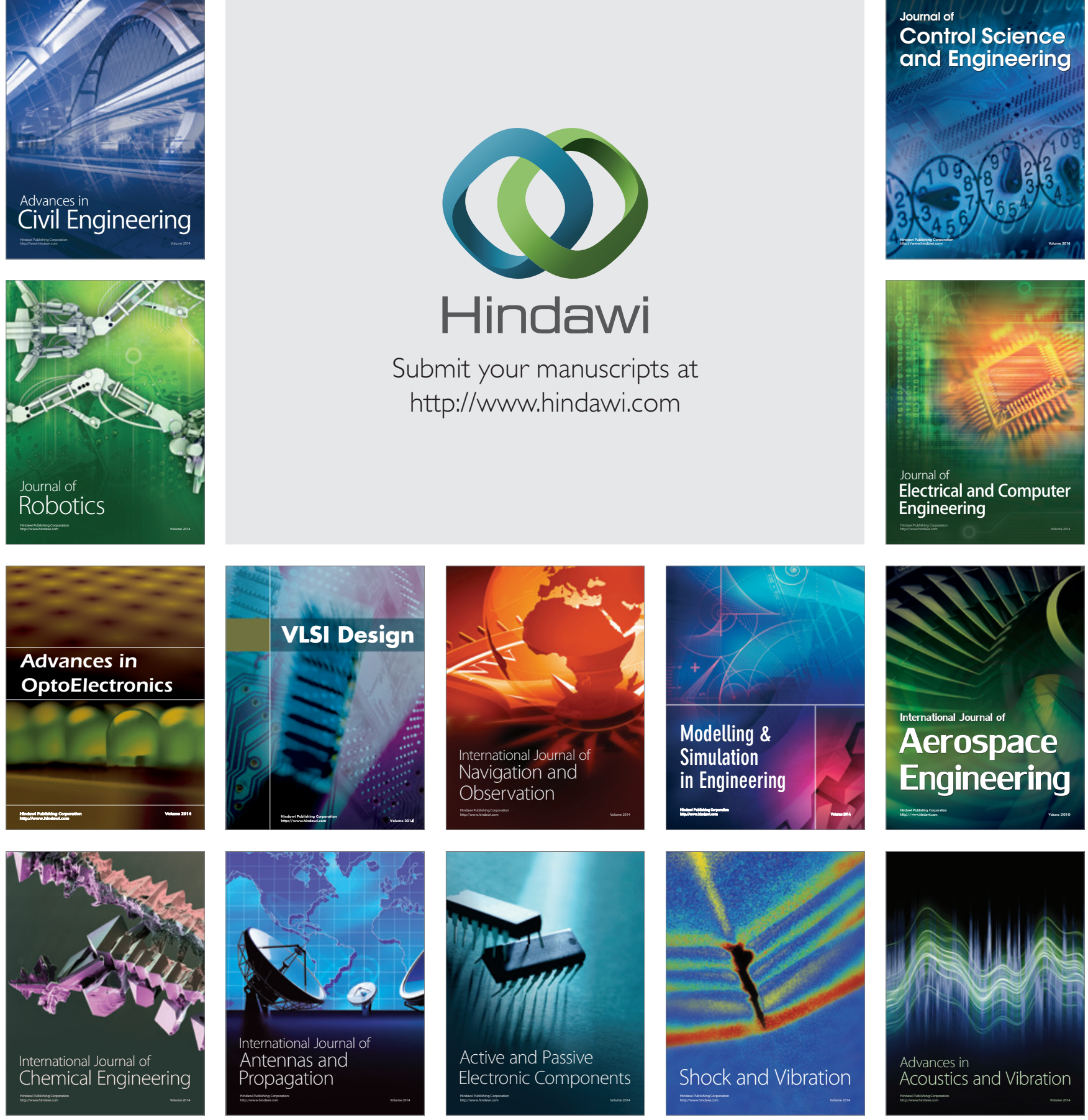